\title{
Morfologia de plântulas de Martiodendron excelsum e sua relevância sistemática em Dialiinae (Leguminosae, "Caesalpinioideae")
}

Seedling morphology of Martiodendron excelsum and its systematic relevance in Dialiinae (Leguminosae, "Caesalpinioideae")

\author{
Leonardo da Silva Hartmann ${ }^{1} \&$ Rodrigo Schütz Rodrigues ${ }^{1,2}$
}

\begin{abstract}
Resumo
Martiodendron Gleason é um gênero sul-americano com cinco espécies arbóreas, subordinado à Dialiinae (tribo Cassieae). Dialiinae apresenta 17 gêneros, que formam um clado cujas relações genéricas permanecem em grande parte não definidas. Este trabalho descreve e ilustra o desenvolvimento e a morfologia de plântulas de Martiodendron excelsum e avalia, por meio de uma revisão, a relevância sistemática dos atributos de plântulas em nível de gênero em Dialiinae. Sementes de M. excelsum foram coletadas de populações em áreas de mata ciliar no Estado de Roraima, Brasil. Martiodendron excelsum tem plântulas fanero-epígeoarmazenadoras, eofilos estipulados e 1-foliolados nos três primeiros nós. Os dados sobre morfologia de plântulas são potencialmente úteis para diagnosticar gêneros em Dialiinae, visto que Martiodendron e todos os demais gêneros podem ser distintos entre si pela a variação no grupo morfológico de plântulas, na filotaxia e número de folíolos no primeiro e segundo eofilos, nas relações entre o comprimento e largura dos cotilédones e entre o comprimento do hipocótilo e epicótilo e no alongamento do primeiro entrenó eofilar. Por fím, em Leguminosae, a predominância de eofilos 1-foliolados na maior parte dos gêneros de Dialiinae é somente comparável à encontrada entre as plântulas de táxons de Papilionoideae.
\end{abstract}

Palavras-chave: Cassieae, desenvolvimento pós-seminal, Roraima, taxonomia.

\begin{abstract}
Martiodendron Gleason is a South American genus with five species of trees, belonging to the tribe Cassieae, subtribe Dialiinae (Leguminosae, "Caesalpinioideae"). Dialiinae has 17 genera that form a clade whose generic relationships are not highly resolved. This paper describes and illustrates the early development and seedling morphology of Martiodendron excelsum. We review the current state of knowledge of seedling morphology in Dialiinae, in order to evaluate its systematic relevance at a generic level. Seeds of $M$. excelsum were collected from wild populations in Roraima State, Brazil. Martiodendron excelsum has phanerocotylar, epigeal seedlings with storage cotyledons, with stipulate and 1-foliolate eophylls at the first three nodes. Seedling morphology data are potentially useful for diagnosing genera in Dialiinae. Martiodendron and all other remaining genera can be distinguished from each other by the variation in seedling morphological groups, as well as lengthwidth ratio of cotyledons, phyllotaxis and leaflet number of the first and second eophylls, ratio of hypocotyl length to epicotyl length, and elongation of the first eophyllar internode. In addition, within Leguminosae, the predominance of 1-foliolate eophylls in most genera of Dialiinae is only comparable among seedlings of Papilionoideae taxa.
\end{abstract}

Key words: Cassieae, post-seminal development, Roraima, taxonomy.

\section{Introdução}

$\mathrm{Na}$ subfamília "Caesalpinioideae" (Leguminosae), o gênero sul-americano Martiodendron Gleason está subordinado à tribo Cassieae, subtribo Dialiinae (Bruneau et al. 2008). O gênero apresenta cinco espécies arbóreas (Koeppen \& Iltis 1962; Lombardi 2002), todas encontradas no Brasil (Lima 2013), das quais três ocorrem na Amazônia brasileira (Silva et al. 2005).

\footnotetext{
${ }^{1}$ Universidade Federal de Roraima, Centro de Estudos da Biodiversidade, Av. Cap. Ene Garcez 2413, Aeroporto, 69310-000, Boa Vista, RR, Brasil.

${ }^{2}$ Autor para correspondência: rodrigo.schutz@ufrr.br
} 
Uma das espécies amazônicas é Martiodendron excelsum (Benth.) Gleason. Conhecida popularmente como "tapioqueira", é uma árvore que alcança até 30 metros de altura, com folhas imparipinadas e folíolos alternos. Suas flores apresentam corola amarelo-dourada e os frutos são samaroides (Fig. 1a-b). As principais características que permitem distinguir $M$. excelsum das demais espécies do gênero são o ovário glabro lateralmente e o fruto com alas com cerca de $5 \mathrm{~mm}$ de comprimento (Fig. 1c) (Koeppen \& Iltis 1962; Silva et al. 2005). Martiodendron excelsum ocorre principalmente em matas ciliares no Brasil (estados de Roraima, Amazonas e Pará) e na República Cooperativa da Guiana (Koeppen \& Iltis 1962; Silva et al. 2005; Lima 2013).

Dialiinae conta com 17 gêneros (Lewis 2005), que formam um clado cujas relações genéricas permanecem em grande parte não definidas (LPWG 2013). As relações filogenéticas de Martiodendron, ainda controversas na literatura, exemplificam as atuais imprecisões no conhecimento sobre Dialiinae. Alguns caracteres da anatomia do lenho aproximam Martiodendron com Androcalymma Dwyer, um gênero amazônico monoespecífico (Koeppen \& Iltis 1962). Por outro lado, uma análise cladística com base em caracteres morfológicos e moleculares, sem amostrar Androcalymma, indicou a formação de um clado entre Martiodendron e Koompassia Maingay ex Benth. (Herendeen et al. 2003). A presença de alas vascularizadas nos frutos seria uma possível sinapormofia do clado
Martiodendron-Koompassia (Herendeen et al. 2003), este último um gênero com três espécies ocorrentes no Sudeste Asiático (Lewis 2005). Posteriormente, Bruneau et al. (2008), em uma análise cladística utilizando três marcadores moleculares, encontraram Martiodendron como grupo irmão de Zenia Chun, gênero monoespecífico ocorrente na China e Vietnã. Nesta análise (Bruneau et al. 2008), Koompassia foi considerado grupo-irmão de Mendoravia Capuron, gênero monoespecífico endêmico de Madagascar (Lewis 2005). Contudo, em ambos os casos, estas relações receberam um fraco suporte, destacando-se que Androcalymma também não foi incluído na análise (Bruneau et al. 2008).

O LPWG (2013) enfatiza a necessidade de se integrarem informações provenientes de diferentes caracteres morfológicos aos estudos filogenéticos moleculares para, entre outras razões, melhorar o diagnóstico e a resolução dos clados em Leguminosae. Neste sentido, estudos morfológicos sobre a morfologia de plântulas podem fornecer caracteres com significado taxonômico (e.g. Lima 1990; López et al. 1998; Rodrigues \& Tozzi 2007a,b; 2008, Gurgel et al. 2012; Rodrigues et al. 2012) ou filogenético em Leguminosae (Rodrigues \& Tozzi 2007c; Cardoso et al. 2012).

Para o clado Dialiinae (sensu Bruneau et al. 2008), existem dados sobre a morfologia de plântulas para Apuleia Mart., Dialium L., Dicorynia Benth., Distemonanthus Benth., Koompassia, Labichea Gaudich. ex DC., Petalostylis R. Br.
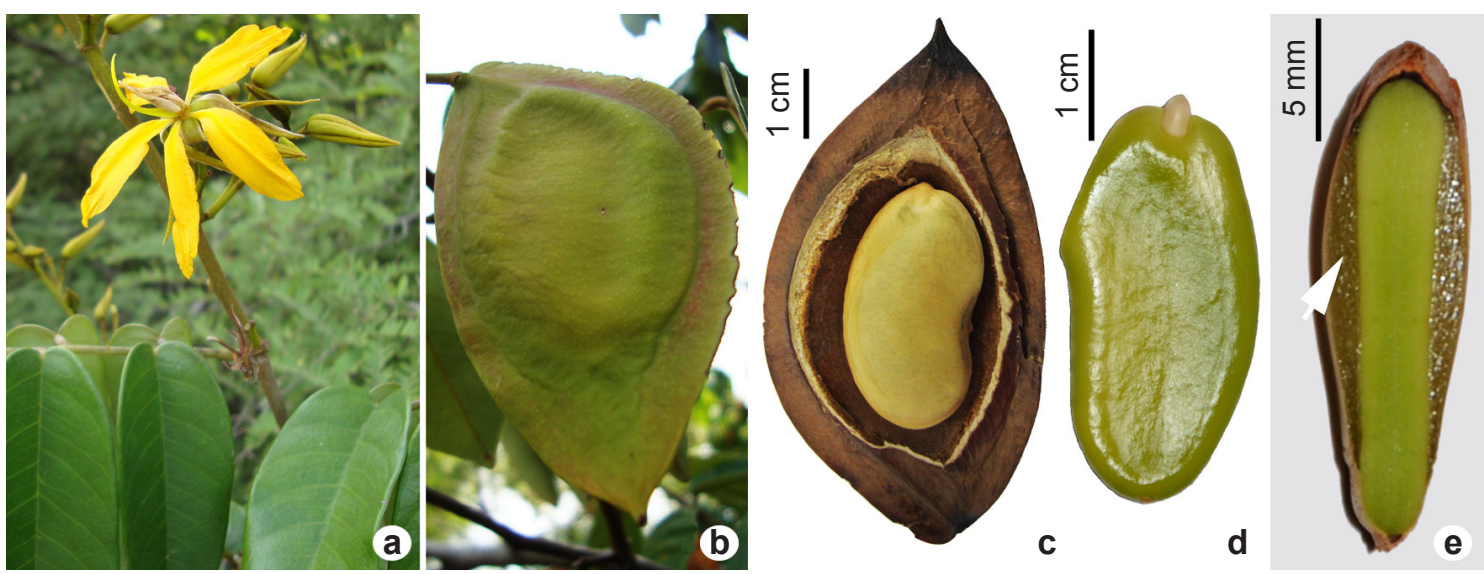

Figura 1 - a-e. Martiodendron excelsum (Benth.) Gleason - a. ramo florífero; b. fruto imaturo; c. fruto maduro em secção longitudinal, mostrando a semente única; d. embrião; e. semente em secção longitudinal, seta indicando o endosperma gelatinoso (L.S. Hartmann 17).

Figure 1 - a-e. Martiodendron excelsum (Benth.) Gleason - a. flowering branch; b. immature fruit; c. mature fruit in longitudinal section, showing a single seed; d. embryo; e. seed in longitudinal section, arrow indicating the gelatinous endosperm (L.S. Hartmann 17). 
e Storckiella Seem. (Tab. 1). Entretanto, para as espécies de Martiodendron não existem até o presente momento informações na literatura sobre sua morfologia de plântulas.

Desta forma, o presente trabalho visa descrever e ilustrar o desenvolvimento e a morfologia de plântulas de Martiodendron excelsum e determinar, principalmente em nível de gênero, a relevância sistemática dos atributos do desenvolvimento inicial em Dialiinae.

\section{Material e Métodos}

O trabalho de campo ocorreu em área de mata ciliar localizada no Campus do Cauamé da Universidade Federal de Roraima, Boa Vista, Roraima, Brasil (Flores \& Rodrigues 2010). A área de coleta está inserida na ecorregião das "Savanas das Guianas" (Capobianco et al. 2001), conhecida regionalmente como "lavrados" (Barbosa et al. 2007). A coleta dos frutos de Martiodendron excelsum e a localização de suas plântulas em ambiente natural ocorreram entre os meses de março a outubro de 2012. Os materiais testemunhos foram depositados nos herbários do Museu Integrado de Roraima (MIRR) e da Universidade Federal de Roraima (UFRR): planta adulta - L.S. Hartmann 17 (MIRR, UFRR); plântulas - L.S. Hartmann 04 (UFRR), 05 (UFRR), 06 (MIRR) e 13 (MIRR).

Os estudos do desenvolvimento e morfologia da plântula de Martiodendron excelsum se deram em condições de laboratório e casa de vegetação.
Sementes foram retiradas manualmente dos frutos, lavadas em água corrente e escarificadas mecanicamente por meio de lâmina. Posteriormente, as sementes foram acondicionadas em caixas de germinação (Gerbox), sob uma camada de algodão e papel filtro, umedecidas com água destilada e mantidas em temperatura ambiente $\left(\right.$ ca. $25^{\circ} \mathrm{C}$ ) em laboratório. Após a germinação, as plântulas foram transferidas para tubetes de plástico com substrato de terra preta e areia de $1 \times 1$, para continuarem o seu desenvolvimento até a formação completa do primeiro par de eofilos no laboratório. A seguir, as plântulas foram transferidas para Estufa de Vegetação do CBio-UFRR para continuar seu desenvolvimento, com sombrite de $75 \%$. Complementarmente, um lote de 13 sementes foi semeado em condições naturais, sem retirá-las do interior das sâmaras, utilizando tubetes de plástico com substrato e condições de estufa de vegetação idênticas ao tratamento anterior.

O conceito de plântula foi adotado segundo Parra (1984), que a considera desde a germinação até o aparecimento do metafilo. Os dados qualitativos e quantitativos foram tomados somente até o completo desenvolvimento do terceiro eofilo, de acordo com Rodrigues \& Tozzi (2008). Descrições foram baseadas principalmente em espécimes vivos, sendo estudadas plântulas de 25 indivíduos de Martiodendron excelsum. As dimensões das estruturas foram tomadas com paquímetro analógico. Para a observação morfológica foi

Tabela 1 - Fontes de dados comparativos sobre morfologia de plântulas de gêneros de Dialiinae (Leguminosae, "Caesalpinioideae").

Table 1 -Sources of comparative data for seedling morphology of Dialiinae genera (Leguminosae, "Caesalpinioideae").

\begin{tabular}{lll}
\hline Táxon & Referência & Distribuição \\
\hline Apuleia leiocarpa (Vogel) J.F.Macbr. & Salazar \& Soihet (2001); Garwood 2009; Felippi et al. (2012) & América do Sul \\
Dialium guianense (Aubl.) Sandwith & Garwood (2009) & Neotropical \\
$\begin{array}{l}\text { D. guineense Willd. } \\
\text { D. platysepalum Baker }\end{array}$ & Brunken et al. (2008) & África \\
Distemonanthus benthamianus Baill. & Miquel (1987); Doucet et al. (2007); Owusu \& Louppe (2012) & África \\
Dicorynia guianensis Amshoff & Jesel (2005); Baraloto \& Forget (2007) & América do Sul \\
Koompassia excelsa (Becc.) Taub. & de Vogel (1980); $\mathrm{Ng}(2013 b)$ & Ásia \\
K. malaccensis Maingay ex Benth. & de Vogel (1980); Ng (2013c) & Ásia \\
Labichea nitida Benth. & Hyland et al. (2010) & Austrália \\
Martiodendron excelsum (Benth.) Gleason & presente trabalho & América do Sul \\
Storckiella australiensis J.H.Ross \& B.Hyland & Hyland et al. $(2010)$ & Austrália \\
Petalostylis labicheoides R.Br. & Compton (1912) & Austrália \\
\hline
\end{tabular}


empregado microscópio estereoscópico Nikon SMZ800, com câmara clara acoplada e os registros fotográficos feitos com máquina Nikon D90.

A terminologia morfológica foi baseada em de Vogel (1980), Rodrigues \& Tozzi (2008) e Garwood (2009). A classificação das plântulas utilizada seguiu Garwood (2009), que emprega três características morfológicas dos cotilédones (exposição, posição e textura), gerando cinco tipos morfofuncionais diferentes (siglas originais em inglês): PEF (fanero-epígeo-foliáceo), PER (faneroepígeo-armazenador), PHR (fanero-hipógeoarmazenador), CER (cripto-epígeo-armazenador) e CHR (cripto-hipógeo-armazenador). Por sua vez, os eofilos do primeiro, segundo e terceiro nós eofilares são abreviados como E1, E2 e E3 respectivamente.

\section{Resultados}

Desenvolvimento da plântula

de Martiodendron excelsum

Os frutos de Martiodendron excelsum são unisseminados (Fig. 1c), e suas sementes apresentam embrião com cotilédones verdes e eixo hipocótilo-radícula alvo e ligeiramente oblíquo em relação aos cotilédones (Fig. 1d), envolto por endosperma gelatinoso (Fig. 1e).

Em condições de laboratório, a germinação das sementes ocorreu cerca de dois a oito dias após a semeadura (Fig. 2a), com o rompimento da testa pelo eixo hipocótilo-radícula junto à região do hilo. Cerca de seis dias após a germinação, observa-se a elongação do hipocótilo e da raiz primária (Fig. 2b). A seguir, ocorre o erguimento da semente acima do nível do solo (Fig. 2c) e, por volta de oito a 10 dias, se dá a exposição dos cotilédones, através do descarte da testa. Aos 15 dias após a germinação (Fig. 2d) o epicótilo começa a se alongar.

Posteriormente, com a completa expansão do epicótilo, o primeiro par de eofilos se alonga (Fig. 2e). Inicialmente verdes, os primeiros eofilos tornamse marrom-avermelhados. Nesse estádio, por volta de 25 dias após a germinação (Fig. 3a-b), o pecíolo e a lâmina dos eofilos expandem-se completamente e gradativamente os eofilos vão passando da coloração marrom-avermelhada para verde. Entre a expansão completa do primeiro par de eofilos e a formação do eofilo do segundo nó, os cotilédones iniciam sua senescência: progressivamente se adelgaçam, tornam-se amarelados e por fim se desprendem da plântula, deixando uma cicatriz cotiledonar. Por volta de 38 dias, tem-se a formação completa do segundo eofilo, apresentando morfologia similar à do primeiro par de eofilos. Aos 46 dias, a plântula exibe seu terceiro eofilo expandido, também similar aos eofilos anteriores. Neste estádio (Fig. 3c), a plântula mantêm sua pubescência no epicótilo, estípulas, pecíolo (Fig. 3d) e face abaxial da lâmina dos eofilos, o hipocótilo adquire uma coloração marrom e as raízes secundárias são relativamente numerosas.

A germinação de sementes de Martiodendron excelsum em condições naturais ocorreu através da emissão do eixo hipocótilo-radícula, rompendo inicialmente o pericarpo (Fig. 4a). Isto iniciou 41 dias após a semeadura e se prolongou por cerca de 90 dias. Logo após, com a fixação da raiz primária no solo, teve início o alongamento do hipocótilo, que elevou os cotilédones juntamente com o pericarpo acima do solo. As calosidades encontradas na base de cada cotilédone desempenharam um papel importante no desenvolvimento da plântula, pois proporcionaram apoio mecânico para permitir a exposição dos cotilédones (Fig. 4b). A exposição de cada cotilédone se deu sequencialmente e, como também observada no ambiente natural, se completou após a expansão total do primeiro par de eofilos (Fig. 4c-d).

Morfologia da plântula

de Martiodendron excelsum

Plântula fanero-epígeo-armazenadora (PER). Raiz primária 170-190 × 2-3 mm, raízes secundárias abundantes, castanhas, não tuberizadas. Hipocótilo $22-56 \times 2,5-3,8 \mathrm{~mm}$, cilíndrico, marrom, glabro. Cotilédones 32,5-46 × 16-23 × 2-3 mm, sésseis, reniformes, plano-convexos, verdes, glabros em ambas as faces, 3-nervados, nervuras adaxiais impressas, as abaxiais sulcadas, base sagitada, adaxialmente com uma calosidade em cada cotilédone, ápice arredondado. Epicótilo 87-148 $\times 2-3 \mathrm{~mm}$, cilíndrico, verde; densamente longopubescente, com tricomas canescentes que também recobrem subsequentemente as demais partes vegetativas. Entrenó-1 4-25 mm compr., entrenó-2 5-17 mm compr., gemas axilares conspícuas. Estípulas 3-7 mm compr., livres entre si, lanceoladas. Eofilos com lâminas foliolares ovadas, face adaxial glabra, abaxial pubescente, ápice acuminado, base cordada, margem inteira, nervação broquidódroma; nictinastia descendente. Eofilos do $1^{\circ}$ nó (E1) opostos, 1-foliolados, pecíolo 12-27 mm compr., lâmina 50-84,5 × 33-72 mm compr.; eofilos do $2^{\circ}$ nó (E2) alternos, 1-foliolados, pecíolo 6-21,5 mm compr., lâmina 31,5-91 × 19-61 mm compr.; eofilos do $3^{\circ}$ nó (E3) alternos, 1-foliolados, pecíolo 8-21 mm compr., lâmina 43-80,5 × 27-52 mm compr. 

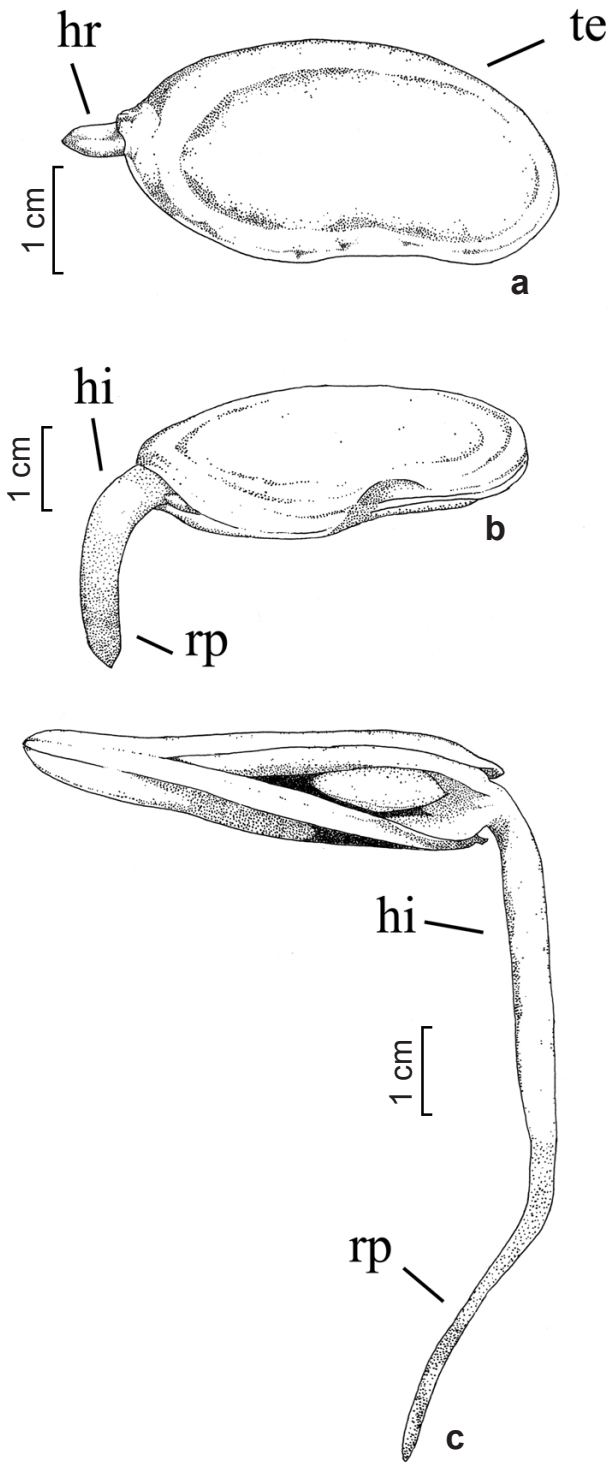

te

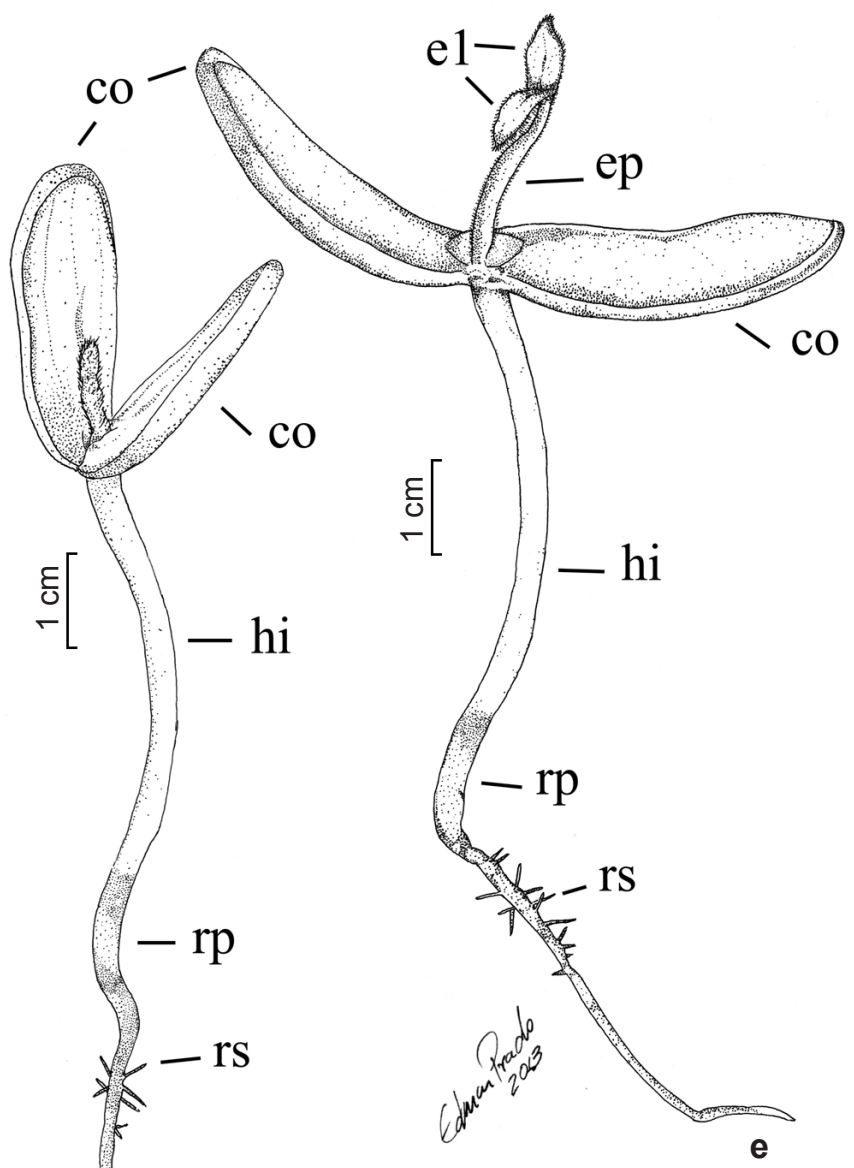

Figura 2 - a-e. Desenvolvimento inicial da plântula de Martiodendron excelsum (Benth.) Gleason - a. com 2 dias; b. com 6 dias; c. com 10 dias; d. com 15 dias; e. com 20 dias. co= cotilédone; e1= eofilo do primeiro nó; ep=epicótilo; hi= hipocótilo; hr= eixo hipocótilo-radícula; $\mathrm{rp}=$ raiz primária; $\mathrm{rs}=$ raiz secundária; te= testa (L.S. Hartmann 06). Figure 2 - a-e. Initial development of the seedling of Martiodendron excelsum (Benth.) Gleason - a. 2 days old; b. 6 days old; c. 10 days old; d. 15 days old; e. 20 days old. co= cotyledon; e1= eophyll at first node; ep= epicotyl; hi= hypocotyl; hr= hypocotyl-radicle axis; $\mathrm{rp}=$ primary root; $\mathrm{rs}=$ secondary root; te $=$ testa (L.S. Hartmann 06$)$.

\section{Discussão}

Martiodendron excelsum tem suas plântulas descritas aqui pela primeira vez, o que também representa um registro inédito para o gênero.

Com relação ao desenvolvimento das plântulas, M. excelsum apresenta cotilédones armazenadores, que inicialmente atuam como haustoriais, pela presença de endosperma (Smith 1983; Garwood
1996). Além disso, em Martiodendron, existia controvérsia na literatura sobre a presença de endosperma. Gunn (1991) afirma que as sementes de Martiodendron seriam exalbuminosas. Entretanto, nossas observações concordam com Koeppen \& Iltis (1962), Oliveira \& Pereira (1984) e Silva et al. (2005), que também relatam a ocorrência de endosperma gelatinoso em Martiodendron. 


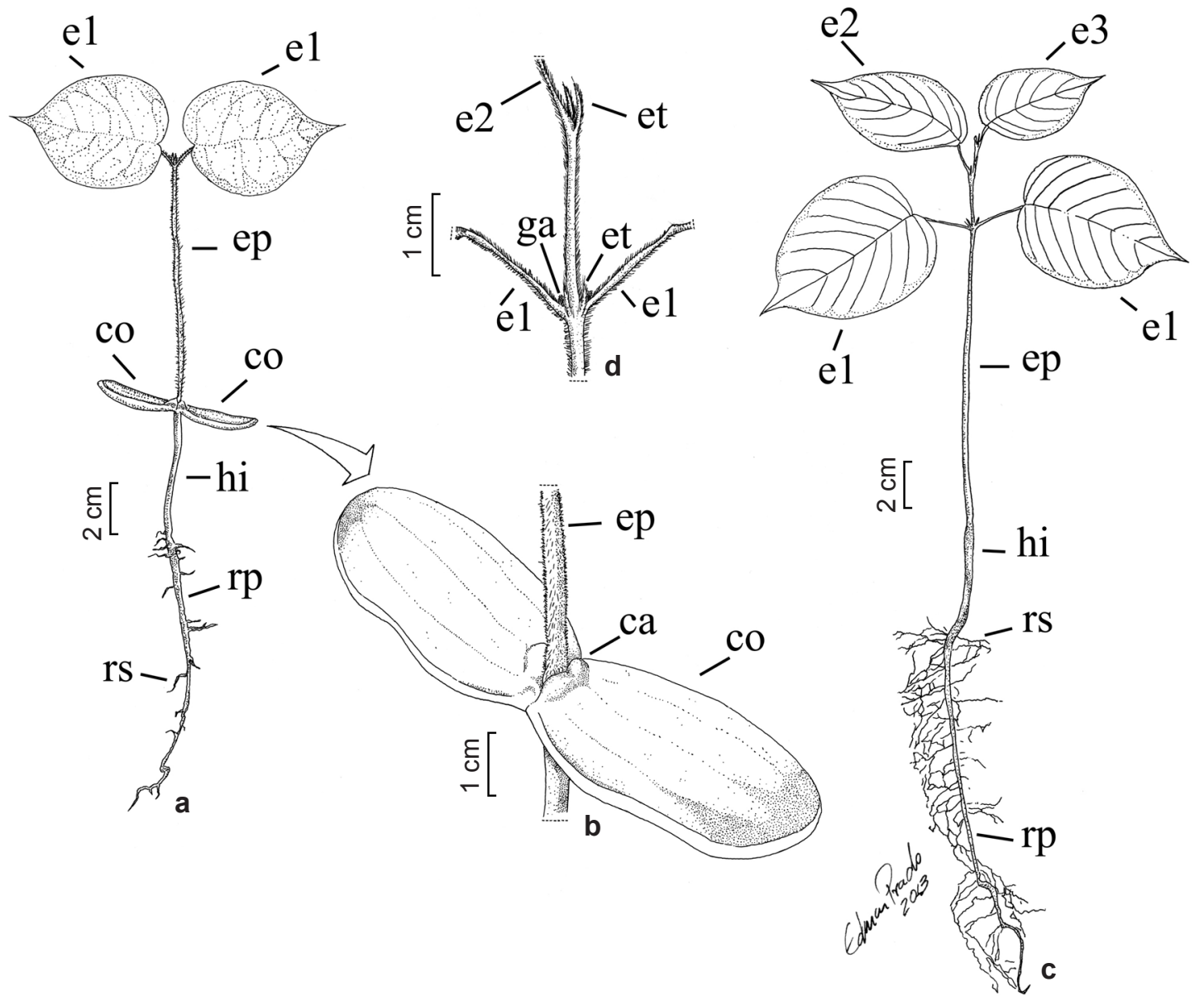

Figura 3 - a-d. Plântula de Martiodendron excelsum (Benth.) Gleason - a. com 25 dias; b. detalhe dos cotilédones, adaxialmente com calosidades; c. com 46 dias; d. detalhe do primeiro entrenó eofilar expandido, evidenciando as gemas axilares conspícuas no primeiro nó eofilar e as estípulas nos dois primeiros eofilos. ca $=$ calosidade; $c o=$ cotilédone; e1= eofilo do primeiro nó; $\mathrm{e} 2=$ eofilo do segundo nó; e3= eofilo do terceiro nó; ep= epicótilo; et= estípula; ga= gema axilar; hi= hipocótilo; $\mathrm{rp}=$ raiz primária; $\mathrm{rs}=$ raiz secundária (L.S. Hartmann 13).

Figure 3 - a-d. Seedling of Martiodendron excelsum (Benth.) Gleason - a. 25 days old; b. detail of the adaxially callous cotyledons; c. 46 days old; d. detail of the expanded first eophyllar internode, showing conspicuous axillary buds at the first eophyllar node, and stipules of the first two eophylls. $\mathrm{ca}=$ callus; $\mathrm{co}=$ cotyledon; e1= eophyll at first node; e2= eophyll at second node; e3=eophyll at third node; ep= epicotyl; et= stipule; ga= axillary bud; hi= hypocotyl; rp= primary root; rs= secondary root (L.S. Hartmann 13).

A revisão da literatura, em conjunção com os dados deste trabalho, indica que existem informações sobre a morfologia de plântulas de nove gêneros de Dialiinae, o que representa cerca da metade de seus 17 gêneros (Bruneau et al. 2008). Algumas características da morfologia de plântulas, ou a sua combinação, podem ser diagnósticas em nível genérico em Dialiinae (Tab. 2) e alguns exemplos são discutidos abaixo.

Na análise de Bruneau et al. (2008), Labichea foi reconhecido como grupo irmão do clado formado por Storckiella e Petalostylis. Labichea apresenta cotilédones elípticos e E1 1-foliolados (Hyland et al. 2010). Entretanto, sua característica mais distintiva são os E2 5-foliolados, pinados, cujo folíolo terminal é nitidamente maior que os demais (Hyland et al. 2010). Por outro lado, Storckiella apresenta cotilédones largo-obovados, mais largos do que longos, e os E1 e E2 1-foliolados (Hyland et al. 2010). Os dados sobre as plântulas de Petalostylis ainda são incompletos, mas seus cotilédones foliáceos e elípticos (Compton 1912; Smith 1983) podem auxiliar na sua distinção de Storckiella. 
Apuleia, Dialium, Dycorinia e Martiodendron apresentam representantes neotropicais cujas plântulas foram estudadas, no mínimo, em uma espécie de cada gênero. Estes quatro gêneros apresentam plântulas PER, cotilédones reniformes a elípticos e E1 e E2 1-foliolados. Destes, Dialium é o único gênero anfi-atlântico (Lewis 2005). As plântulas das espécies estudadas de Dialium possuem hipocótilo mais longo ou do mesmo comprimento que o epicótilo. Contudo, a principal característica distintiva das plântulas de Dialium é o primeiro entre-nó eofilar marcadamente curto, tornando E1 e E2 relativamente próximos entre si ( $\mathrm{Ng} 1978,2013 \mathrm{a}$; Brunken et al. 2008; Garwood 2009).

Análises morfológicas e moleculares têm agrupado Dycorinia dentro de um clado com algumas espécies de Dialium (Herendeen et al. 2003; Bruneau et al. 2008). Contudo, Dycorinia apresenta o primeiro entre-nó eofilar alongado, não marcadamente curto (Jesel 2005), o que também é encontrado em Apuleia e Martiodendron. Dycorynia e Apuleia apresentam hipocótilo mais longo que o epicótilo e E1 opostos ou alternos, respectivamente. Desta forma, Martiodendron distingue-se dos dois gêneros anteriores por apresentar hipocótilo mais curto que o epicótilo.

Estudos moleculares têm mostrado Apuleia como grupo-irmão de Distemonanthus (Bruneau et al. 2008). As principais diferenças da morfologia de plântulas entre estes gêneros são as plântulas PEF e o epicótilo fortemente reduzido em Distemonanthus (Miquel 1987; Doucet et al. 2007; Owusu \& Louppe 2012), contrastando com as plântulas PER e o epicótilo relativamente desenvolvido de Apuleia (Salazar \& Soihet 2001; Garwood 2009; Felippi et al. 2012).

Com relação às relações filogenéticas de Martiodendron em Dialiinae, análises morfológicas e moleculares têm indicado possíveis afinidades com Androcalymma (Koeppen \& Iltis 1962),
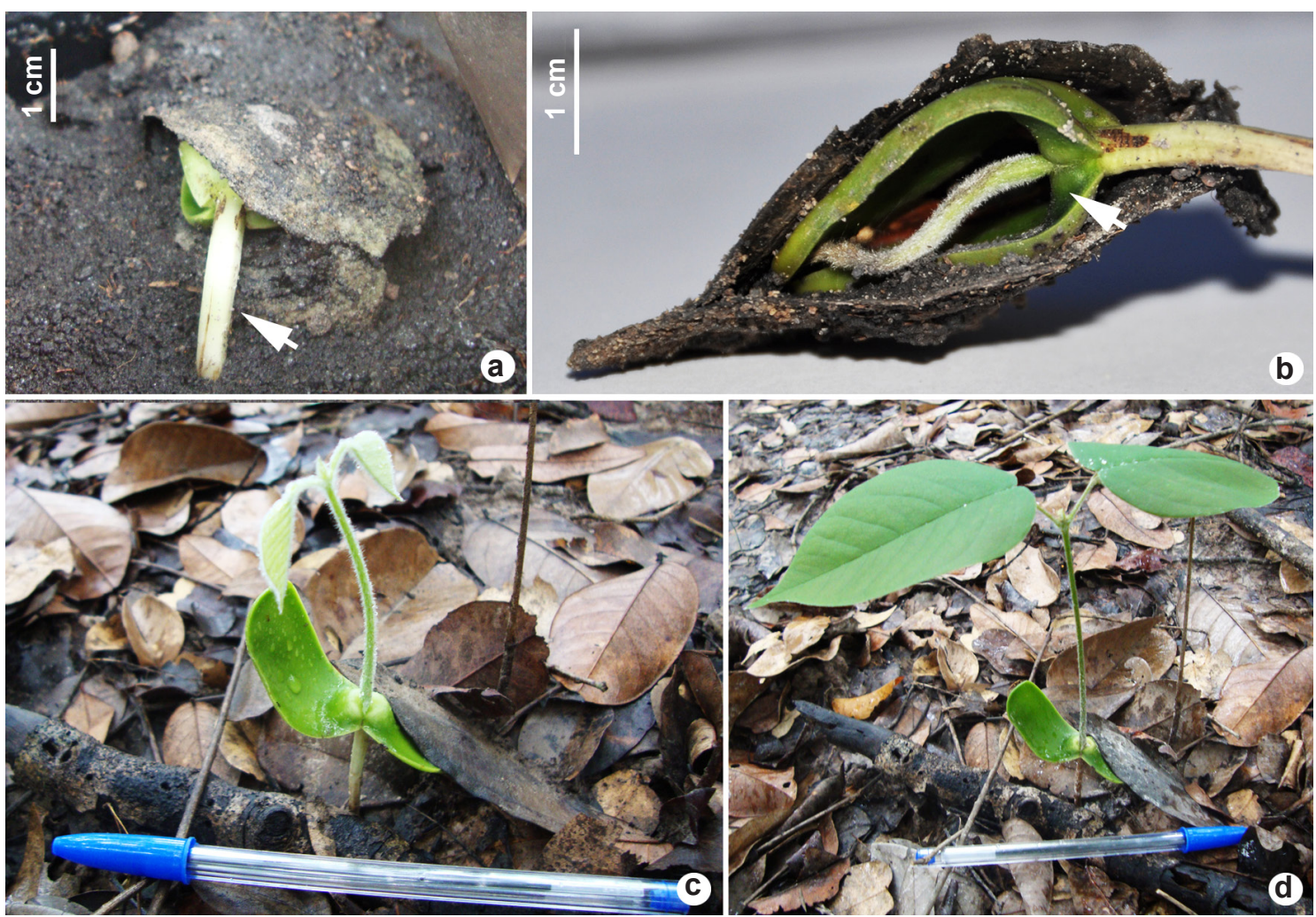

Figura 4 - a-d. Plântula de Martiodendron excelsum (Benth.) Gleason - a. emissão do eixo hipocótilo-radícula, rompendo o pericarpo; b. corte longitudinal do fruto, evidenciando as calosidades adaxiais dos cotilédones (seta); c-d. plântulas no ambiente natural, em mata ciliar nas savanas de Roraima, Brasil.

Figure 4 - a-d. Seedling of Martiodendron excelsum (Benth.) Gleason - a. emerging of the hypocotyl-radicle axis, breaking the pericarp; b. Fruit in longitudinal section, showing the adaxially callous cotyledons (arrow); c-d. seedlings in the natural habitat, a riparian forest in Roraima savannas, Brazil. 
Tabela 2 - Sumário das principais características diagnósticas da morfologia de plântulas em gêneros de Dialiinae (Leguminosae, "Caesalpinioideae"). Plânt. = grupo morfológico de plântula; PER= plântula fanero-epígeoarmazenadora; $\mathrm{PEF}=$ plântula fanero-epígeo-foliácea; Cotil.= cotilédone; $\mathrm{E} 1=$ eofilo do primeiro nó; E2= eofilo do segundo nó; $\mathrm{C}=$ comprimento; $\mathrm{L}=$ largura; fol. = folíolo; alt.= alterno; op.= oposto; Hip.= hipocótilo; Ep.= epicótilo; s.d. $=$ sem dados disponíveis.

Table 2 - Summary of the most important diagnostic seedling morphology characters in Dialiinae genera (Leguminosae, "Caesalpinioideae"). Plânt.= seedling morphology group; PER= phanerocotylar, epigeal, reserve seedling; PEF= phanerocotylar, epigeal, foliaceous seedling; Cotil.= cotyledon; E1= first node eophyll; E2= second node eophyll; $\mathrm{C}=$ length; $\mathrm{L}=$ width; fol. = leaflet; alt.= alternate; op. = opposite; Hip.= hypocotyl; Ep.= epicotyl; s.d.= data not available.

\begin{tabular}{|c|c|c|c|c|c|c|}
\hline Gênero & Plânt. & Cotil. & E1 & $\mathbf{E 2}$ & Hip./Ep. & Observação \\
\hline Apuleia & PER & $\mathrm{C}>\mathrm{L}$ & 1-fol.; alt. & 1-fol.; alt. & hip.>ep. & - \\
\hline Dialium & PER & $\mathrm{C}>\mathrm{L}$ & 1-fol.; op. & 1-fol.; op. & hip. $\geq$ ep. & entre-nó eofilar reduzido \\
\hline Distemonanthus & PEF & $\mathrm{C}>\mathrm{L}$ & 1-fol.; op. & 1-fol.; alt. & hip.>ep. & ep. reduzido \\
\hline Dycorinia & PER & $\mathrm{C}>\mathrm{L}$ & 1-fol.; op. & 1-fol.; alt. & hip.>ep. & - \\
\hline Koompassia & PER & $\mathrm{C}>\mathrm{L}$ & 8-11 fol.; op. & 8-11-fol., alt. & hip. $\leq$ ep. & - \\
\hline Labichea & PER & $\mathrm{C}>\mathrm{L}$ & 1-fol., alt. & 5-fol.; alt. & hip. > ep. & - \\
\hline Martiodendron & PER & $\mathrm{C}>\mathrm{L}$ & 1-fol.; op. & 1-fol.; alt. & hip.< ep. & - \\
\hline Petalostylis & PEF & $\mathrm{C}>\mathrm{L}$ & s.d. & s.d. & s.d. & - \\
\hline Storckiella & PER & $\mathrm{C}<\mathrm{L}$ & 1-fol.; op. & 1-fol.; alt. & hip.< ep. & cotil. largo-obovado \\
\hline
\end{tabular}

Koompassia (Herendeen et al. 2003), ou Zenia (Bruneau et al. 2008). Não foi possível encontrar informação sobre morfologia de plântulas de Androcalymma e Zenia, sendo que o primeiro nunca foi coletado em fruto (Lewis 2005) e já pode estar extinto na natureza (LPWG 2013). Desta forma, até o momento, comparações são possíveis entre as plântulas de Koompassia e Martiodendron.

Para Koompassia, existem dados para duas de suas três espécies, ocorrentes no sudeste asiático (Ng 1978; de Vogel 1980). Koompassia apresenta plântulas PER e sua principal característica distintiva são seus E1 com 8-11 folíolos (Ng 1978, 2013b; 2013c; de Vogel 1980) em comparação com E1 1-foliolados de Martiodendron. De Vogel (1980) descreve detalhadamente as plântulas de Koompassia excelsa (Becc.) Taub. e menciona a sua grande similaridade com as de K. malaccensis Maingay (Ng 1978; de Vogel 1980). As plântulas de K. excelsa e de Martiodendron excelsum apresentam o hipocótilo glabro e seus cotilédones são sésseis, com margem inteira, base auriculada e ápice arredondado. Além disso, os E1 são opostos e estipulados em ambas as espécies. Entretanto, os cotilédones de $K$. excelsa diferem de $M$. excelsum por serem estipulados e com uma única nervura central, sulcada na face adaxial e inconspícua na face abaxial.
Por fim, em uma revisão sobre a morfologia de plântulas de angiospermas neotropicais, Garwood (2009) reconheceu que em "Caesalpinioideae" predominam plântulas com E1 bi a multifoliolados, sendo raramente encontradas plântulas com E1 1-foliolados. Isto contrasta com o encontrado para os gêneros de Dialiinae, pois E1 1-foliolados são uma característica compartilhada por quase todos os gêneros da subtribo, com exceção de Koompassia, cujas espécies apresentam eofilos multifoliolados.

De fato, em "Caesalpinioideae", plântulas com E1 1-foliolados também foram mencionadas para algumas espécies de Copaifera (Garwood 2009), que faz parte da tribo Detarieae (Mackinder 2005). Desta forma, com exceção de Papilionoideae, onde plântulas com E1 1-foliolados são amplamente encontradas em gêneros pertencentes a diversas tribos (Garwood 2009; Gurgel et al. 2012; Prado \& Rodrigues 2013), nossa revisão aponta que Dialiinae é o outro clado de Leguminosae onde este número de folíolos predomina. Além disso, com exceção de Distemonanthus e Petalostylis, que apresentam plântulas PEF, todos os demais gêneros estudados em Dialiinae apresentam plântulas PER.

Em conclusão, as plântulas de Martiodendron excelsum apresentam várias características concordantes com outros gêneros de Dialiinae. Entretanto, mesmo considerando-se a necessidade 
de se ampliar a amostragem dentro da subtribo, a variação no grupo morfológico de plântulas, número de folíolos no primeiro e segundo eofilos, relações entre o comprimento e largura dos cotilédones e entre o comprimento do hipocótilo e epicótilo e o alongamento do primeiro entrenó eofilar apresentam potencial sistemático para diagnosticar gêneros em Dialiinae. Assim, Martiodendron pode ser reconhecido entre os gêneros já estudados na subtribo pela combinação de suas plântulas PER, hipocótilo mais curto que o epicótilo, E1 e E2 1-foliolados, com o primeiro entre-nó eofilar alongado, não marcadamente curto.

\section{Agradecimentos}

Os autores agradecem à Dra. Andréia Flores, a leitura crítica do manuscrito e apoio no Herbário do Museu Integrado de Roraima; a Edmar da Silva Prado, as ilustrações botânicas; ao Prof. Dr. Frank James Araújo Pinheiro, o auxílio no uso da casa de vegetação e ao CBio/UFRR, as facilidades.

\section{Referências}

Baraloto, C. \& Forget, P.M. 2007. Seed size, seedling morphology, and response to deep shade and damage in neotropical rain forest trees. American Journal of Botany 94: 901-911.

Barbosa, R.I.; Campos, C.; Pinto, F. \& Fearnside, P.M. 2007. The "Lavrados" of Roraima: biodiversity and conservation of Brazil's Amazonian Savannas. Functional Ecosystems and Communities 1: 29-41.

Bruneau, A.; Mercure, M.; Lewis, G.P. \& Herendeen, P.S. 2008. Phylogenetic patterns and diversification in the caesalpinioid legumes. Botany 86: 697-718.

Brunken, U.; Schmidt, M.; Dressler, S.; Janssen, T.; Thiombiano, A. \& Zizka, G. 2008. West African plants - a photo guide. Disponível em $<$ www. westafricanplants.senckenberg.de> . Acesso em 5 julho 2013.

Capobianco, J.P.R.; Veríssimo, A.; Moreira, A.; Sawyer, D.; Santos, I \& Pinto, L.P. 2001. Biodiversidade na Amazônia brasileira: avaliação e ações prioritárias para a conservação, uso sustentável e repartição dos benefícios. Instituto Socioambiental/Estação Liberdade, São Paulo. 540p.

Cardoso, D.; Lima, H.C.; Rodrigues, R.S.; Queiroz, L.P.; Pennington, R.T. Lavin, M. 2012 The Bowdichia clade of Genistoid legumes: phylogenetic analysis of combined molecular and morphological data and a recircumscription of Diplotropis. Taxon 61: 1074-1087.

Compton, R.H. 1912. An investigation of the seedling structure in the Leguminosae. The Journal of the Linnean Society (Botany) 41: 1-122.
De Vogel, E. F. de. 1980. Seedlings of dicotyledons. Centre for Agricultural Publishing and Documentation, Wageningen. 465p.

Doucet, J.L.; Dissaki, A. \& Mengome, A. 2007. Dynamique des peuplements forestiers d'Afrique Centrale: fiches descriptives. Faculté Universitaire des sciences agronomiques, Gembloux. Disponível em < http://data.cameroun-foret.com/ bibliotheque/7810>. Acesso em 9 julho 2013.

Felippi, M.; Maffra, C.R.B.; Cantarelli, E.B.; Araújo, M.M. \& Longhi, S.J. 2012. Fenologia, morfologia e análise de sementes de Apuleia leiocarpa (Vogel) J. F. Macbr. Ciência Florestal (Santa Maria) 22: 477-491.

Flores, A.S. \& Rodrigues, R.S. 2010. Diversidade de Leguminosae em uma área de savana estado de Roraima, Brasil. Acta Botanica Brasilica 24: 175183.

Garwood, N. C. 1996. Functional morphology of tropical tree seedlings. In: Swaine, M.D. (ed.). The ecology of tropical forest tree seedlings. Unesco, Paris. Pp. 59-129.

Garwood, N.C. 2009. Seedlings of Barro Colorado Island and the Neotropics. Comstock Publishing Associates, Ithaca. 645p.

Gunn, C.R. 1991. Fruits and seeds of genera in the subfamily Caesalpinioideae (Fabaceae). United States Department of Agriculture Technical Bulletin 1775: 1-408.

Gurgel, E.S.C.; Santos, J.U.M.; Lucas, F.C.A. \& Bastos, M.N.C. 2012. Morfologia de plântulas de Leguminosae e o potencial sistemático. Rodriguésia 63: 65-73.

Herendeen, P.S.; Bruneau, A. \& Lewis, G.P. 2003. Phylogenetic relationships in caesalpinioid legumes: a preliminary analysis based on morphological and molecular data. In: Klitgaard, K.K. \& Bruneau (eds.). Advances in legume systematics. Royal Botanic Gardens, Kew. Part 10. Pp. 37-62.

Hyland, B.P.M.; Whiffin, T.; Zich, F.A.; Duffy, S.; Gray, B.; Elick, R.; Venter, S. \& Christophel, D. 2010. Australian tropical rainforest plants. Edition 6 . Online version. Disponível em $<$ http://www.anbg. gov.au/cpbr/cd-keys/rfk/index.html>. Acesso em 10 julho 2013.

Jesel, S. 2005. Écologie et dynamique de la régénération de Dicorynia guianensis (Caesalpiniaceae) dans une forêt guyanaise. Tese de Doutorado. L'Institut National Agronomique, Paris-Grignon. 288p.

Koeppen, R. \& Iltis, H.H. 1962. Revision of Martiodendron (Cassieae, Caesalpiniaceae). Brittonia 14: 191-209.

Lewis, G.P. 2005. Tribe Cassieae. In: Lewis, G.; Schrire, B.; Mackinder, B. \& Lock, M. (eds.). Legumes of the world. Royal Botanic Gardens, Kew. Pp. 111-125.

Lima, H.C. 1990. Tribo Dalbergieae (Leguminosae Papilionoideae) - Morfologia dos frutos, sementes 
e plântulas e sua aplicação na sistemática. Arquivos do Jardim Botânico do Rio de Janeiro 30: 1-42.

Lima, H.C. 2013. Martiodendron. In: Lista de espécies da flora do Brasil. Jardim Botânico do Rio de Janeiro. Disponível em <http://floradobrasil.jbrj. gov.br/jabot/floradobrasil/FB23081>. Acesso em 10 julho 2013.

Lombardi, J.A. 2002. Martiodendron fluminense (Leguminosae, Caesalpinioideae), a new species from the Atlantic coast rainforest of Brazil. Brittonia 54: 327-330.

López, J.; Devesa, J. A.; Ruiz, T. \& Ortega-Olivencia, A. 1998. Seedling morphology in Genisteae (Fabaceae) from south-west Spain. Botanical Journal of the Linnean Society 127: 229-250.

LPWG (The Legume Phylogeny Working Group). 2013. Legume phylogeny and classification in the $21^{\text {st }}$ century: Progress, prospects and lessons for other species-rich clades. Taxon 62: 217-248.

Mackinder, B. 2005. Tribe Detarieae. In: Lewis, G.; Schrire, B.; Mackinder, B. \& Lock, M. (eds.). Legumes of the world. Royal Botanic Gardens, Kew. Pp. 69-109.

Miquel, S. 1987. Morphologie fonctionelle de plantules d'especes forestiêres du Gabon. Bulletin du Museum National d'Histoire Naturelle, Section B, Adansonia 9: 101-121.

$\mathrm{Ng}$, F.S.P. 1978. Strategies of establishment in Malayan forest trees. In: Tomlinson, P.B. \& Zimmerman, M. (eds.). Tropical Trees as Living Systems. Cambridge University Press, Cambridge. Pp. 129-162.

Ng, F.S.P. 2013a. Dialium platysepalum Baker. In: Provisional checklist of the vascular plants of Malaysia. Disponível em $<\mathrm{http}$ ://www.chm.frim. gov.my/Data/Species/Flora/Provisional-Checklistof-the-Vascular-Plants-of-Ma/Magnoliopsida/ Fabales/Leguminosae/Dialium-platysepalum. aspx>. Acesso em 23 agosto 2013.

Ng, F.S.P. 2013b. Koompassia excelsa (Becc.) Taub. In: Provisional checklist of the vascular plants of Malaysia. Disponível em <http://www.chm.frim. gov.my/Data/Species/Flora/Provisional-Checklistof-the-Vascular-Plants-of-Ma/Magnoliopsida/ Fabales/Leguminosae/Koompassia-excelsa.aspx $>$. Acesso em 23 agosto 2013.

Ng, F.S.P. 2013c. Koompassia malaccensis Maing. ex Benth. In: Provisional checklist of the vascular plants of Malaysia. Disponível em $<$ http:// www.chm.frim.gov.my/Data/Species/Flora/ Provisional-Checklist-of-the-Vascular-Plantsof-Ma/Magnoliopsida/Fabales/Leguminosae/ Koompassia-malaccensis.aspx $>$. Acesso em 23 agosto 2013.
Oliveira, E.C. \& Pereira, T.S. 1984. Morfologia dos frutos alados em Leguminosae-Caesalpinioideae - Martiodendron Gleason, Peltophorum (Vogel) Walpers, Sclerolobium Vogel, Tachigali Aublet e Schizolobium Vogel. Rodriguésia 36: 35-42.

Owusu, F.W. \& Louppe, D. 2012. Distemonanthus benthamianus Baill. In: Lemmens, R.H.M.J.; Louppe, D. \& Oteng-Amoako, A.A. (eds.). PROTA (Plant Resources of Tropical Africa/Ressources végétales de l'Afrique tropicale). Wageningen University, Wageningen. Disponível em <http://www.prota4u. org/search.asp>. Acesso em 5 julho 2013.

Parra, P. 1984. Estudio de la morfologia externa de plántulas de Calliandra gracilis, Mimosa albida, Mimosa arenosa, Mimosa camporum y Mimosa tenuiflora. Revista de la Faculdad de Agronomia (Maracay) 13: 311-350.

Prado, E.S. \& Rodrigues, R.S. 2013. Morfologia de frutos, sementes e plântulas de Canavalia dictyota Piper (Leguminosae, Papilionoideae). Boletim do Museu Integrado de Roraima 7: 28-34.

Rodrigues, R.S.; Hirt, A.P.M \& Flores, A.S. 2012. Morfologia de plântulas das espécies de Rhynchosia (Leguminosae, Papilionoideae) de Roraima, Brasil. Acta Botanica Brasilica 26: 585-592.

Rodrigues, R.S. \& Tozzi, A.M.G.A. 2007a. Morfologia de plântulas de cinco leguminosas genistóides arbóreas do Brasil (Leguminosae-Papilionoideae). Acta Botanica Brasilica 21: 599-607.

Rodrigues, R.S. \& Tozzi, A.M.G.A. 2007b. Morfologia de plântulas no clado Vatairea (Leguminosae, Papilionoideae). Rodriguésia 58: 221-229.

Rodrigues, R.S. \& Tozzi, A.M.G.A. 2007 c. Morphological analysis and re-examination of the taxonomic circumscription of Acosmium (Leguminosae, Papilionoideae, Sophoreae). Taxon 56: 439-452.

Rodrigues, R.S. \& Tozzi, A.M.G.A. 2008. Systematic relevance of seedling morphology in Acosmium, Guianodendron, and Leptolobium (Leguminosae, Papilionoideae). Brittonia 60: 287-296.

Salazar, R. \& Soihet, C. 2001. Manejo de semillas de 75 especies forestales de América Latina. Vol. II. Centro Agronómico de Investigación y Enseñanza, Turrialba. 156p.

Silva, M.F.; Carreira, L.M.M. \& Souza, L.A.G. 2005. Leguminosas da Amazônia Brasileira -X. Martiodendron Gleason (LeguminosaeCaesalpinioideae). Boletim do Museu Paraense Emílio Goeldi, ser. Ciências Naturais 1: 7-29.

Smith, D.L. 1983. Cotyledon anatomy in the Leguminosae. Botanical Journal of the Linnean Society $86: 325-355$. 